\title{
Erratum to: Combined effects of climatic gradient and domestic livestock grazing on reptile community structure in a heterogeneous agroecosystem
}

\author{
Guy Rotem $^{1,4} \cdot$ Yoni Gavish $^{1} \cdot$ Boaz Shacham $^{2} \cdot$ Itamar Giladi $^{1,3} \cdot$ Amos Bouskila $^{4}$. \\ Yaron Ziv ${ }^{1}$
}

Published online: 2 November 2015

(C) Springer-Verlag Berlin Heidelberg 2015

\section{Erratum to: Oecologia \\ DOI 10.1007/s00442-015-3435-y}

Unfortunately, Table 1 was incorrectly published in the original article. The corrected Table 1 is given below:

The online version of the original article can be found under doi:10.1007/s00442-015-3435-y.

Guy Rotem

rottemguy@gmail.com

1 Spatial Ecology Lab, Department of Life Sciences, Ben-Gurion University of the Negev, P.O.B. 653, 8410501 Beer-Sheva, Israel

2 The National Natural History Collections, The Hebrew University of Jerusalem, 91904 Jerusalem, Israel

3 Mitrani Department of Desert Ecology, Swiss Institute for Dryland Environmental and Energy Research, Jacob Blaustein Institutes for Desert Research, BenGurion University of the Negev, Midreshet Ben-Gurion, 8499000 Beer-Sheva, Israel

4 Behavioral Ecology Lab, Department of Life Sciences, BenGurion University of the Negev, 8410501 Beer-Sheva, Israel 
Table 1 Scientific names and distribution patterns of various species, as well as their abundances and occupancies (number of patches in which a species was observed; in parentheses) in the different land units

\begin{tabular}{|c|c|c|c|c|c|}
\hline Scientific name & Distribution pattern & Galon & Lachish & Dvir & Rahat \\
\hline \multicolumn{6}{|l|}{ AGAMIDAE } \\
\hline Stellagama stellio ssp. & Mediterranean & $11(5)$ & $9(4)$ & $2(2)$ & $1(1)$ \\
\hline Stellagama stellio brachydactyla & Arid & 0 & 0 & $2(1)$ & $17(6)$ \\
\hline Trapelus pallidus & Arid & 0 & 0 & 0 & $18(9)$ \\
\hline \multicolumn{6}{|l|}{ ANGUIDAE } \\
\hline Pseudopus apodus & Mediterranean & $6(4)$ & 0 & 0 & 0 \\
\hline \multicolumn{6}{|l|}{ BOIDAE } \\
\hline Eryx jaculus & Mediterranean & $2(2)$ & $2(1)$ & $2(2)$ & $1(1)$ \\
\hline \multicolumn{6}{|l|}{ COLUBRIDAE } \\
\hline Eirenis rothii & Mediterranean & $11(6)$ & $9(5)$ & $5(3)$ & $4(2)$ \\
\hline Eirenis coronella & Arid & 0 & 0 & 0 & $18(6)$ \\
\hline Rhynchocalamus melanocephalus & Mediterranean & $4(3)$ & 0 & $1(1)$ & 0 \\
\hline Telescopus fallax syriacus & Mediterranean & 0 & $1(1)$ & $1(1)$ & 0 \\
\hline Natrix tessellata & Mediterranean & $3(2)$ & 0 & 0 & 0 \\
\hline Dolichophis jugularis & Mediterranean & $14(6)$ & $9(4)$ & $3(2)$ & 0 \\
\hline Platyceps collaris & Mediterranean & $12(5)$ & $11(5)$ & $4(3)$ & 0 \\
\hline Platyceps rogersi & Arid & 0 & 0 & 0 & $21(7)$ \\
\hline \multicolumn{6}{|l|}{ CHAMAELEONIDAE } \\
\hline Chamaeleo chamaeleon recticrista & Mediterranean & $3(2)$ & $3(2)$ & $1(1)$ & 0 \\
\hline \multicolumn{6}{|l|}{ GEKKONIDAE } \\
\hline Hemidactylus turcicus & Generalist & 0 & $5(3)$ & $27(8)$ & $17(7)$ \\
\hline Ptyodactylus guttatus & Generalist & $9(2)$ & 0 & $21(3)$ & $13(3)$ \\
\hline Stenodactylus sthenodactylus & Arid & 0 & 0 & $21(7)$ & $15(6)$ \\
\hline \multicolumn{6}{|l|}{ LACERTIDAE } \\
\hline Ophisops elegans & Mediterranean & $19(6)$ & $8(4)$ & $3(2)$ & $6(4)$ \\
\hline Mesalina olivieri & Arid & 0 & 0 & $6(3)$ & $16(8)$ \\
\hline Mesalina guttulata & Arid & 0 & 0 & $5(2)$ & $19(3)$ \\
\hline \multicolumn{6}{|l|}{ LAMPROPHIIDAE } \\
\hline Malpolon insignitus & Mediterranean & $2(2)$ & $3(3)$ & 0 & 0 \\
\hline Psammophis schokari & Generalist & 0 & 0 & 0 & $11(5)$ \\
\hline Micrelaps muelleri & Mediterranean & $2(2)$ & 0 & 0 & 0 \\
\hline \multicolumn{6}{|l|}{ LEPTOTYPHLOPIDAE } \\
\hline Leptotyphlops macrorhynchus & Arid & 0 & $2(1)$ & $5(3)$ & $21(6)$ \\
\hline \multicolumn{6}{|l|}{ SCINCIDAE } \\
\hline Chalcides ocellatus & Generalist & $12(4)$ & $4(2)$ & $13(5)$ & $8(3)$ \\
\hline Chalcides guentheri & Mediterranean & $28(9)$ & $35(8)$ & $11(5)$ & 0 \\
\hline Trachylepis vittata & Mediterranean & $125(10)$ & $92(10)$ & $45(7)$ & 0 \\
\hline Eumeces schneideri pavimentatus & Mediterranean & $9(6)$ & $12(7)$ & $4(2)$ & $2(1)$ \\
\hline Eumeces schneideri schneideri & Arid & 0 & 0 & $4(2)$ & $18(5)$ \\
\hline Ablepharus rueppellii & Mediterranean & $62(9)$ & $13(6)$ & $3(3)$ & 0 \\
\hline \multicolumn{6}{|l|}{ TESTUDINIDAE } \\
\hline Testudo graeca & Mediterranean & $11(5)$ & $4(3)$ & $8(3)$ & $6(2)$ \\
\hline \multicolumn{6}{|l|}{ TYPHLOPIDAE } \\
\hline Typhlops vermicularis & Mediterranean & 0 & 0 & $6(4)$ & 0 \\
\hline Rhinotyphlops simoni & Mediterranean & 0 & $3(2)$ & $3(1)$ & $6(3)$ \\
\hline \multicolumn{6}{|l|}{ VIPERIDAE } \\
\hline Daboia palaestinae & Mediterranean & $4(3)$ & $1(1)$ & 0 & 0 \\
\hline
\end{tabular}

Nomenclature follows accepted taxonomic names at The Reptile Database website, http://reptile-database. reptarium.cz/. Accessed 14 September 2015 